\title{
Co-relative mobility based loT data uploading using D2D communication
}

\author{
Sunny Sanyal \\ Chongqing University of Posts \\ and Telecommunications \\ China \\ sanyal.sunny111@ \\ yahoo.com
}

\author{
Dr.Dapeng $\mathrm{Wu}^{\dagger}$ \\ Chongqing University of Posts \\ and Telecommunications \\ China \\ wudp@cqupt.edu.cn
}

\author{
Junjie Yan $^{\ddagger}$ \\ Chongqing University of Posts \\ and Telecommunications \\ China \\ 358638469@qq.com
}

\author{
Xing $\mathrm{Li}^{\S}$ \\ Chongqing University of Posts \\ and Telecommunications \\ China \\ cxcqupt@126.com
}

\begin{abstract}
Fifth generation (5G) systems are imminent and with the increasing data rates, there is consensus among global industries and academia to devise a digital world which is $24 \mathrm{X} 7$ connected. To make this happen we need to rely on our smart devices, cloud-based services and D2D communication as the potential candidates, however, since we are focusing on the wireless domain, where energy still remains a key constraint in the development. We propose a framework, whose application can be extended to IEEE 802.15.4 protocol, i.e. low power devices (IoT devices). Our idea is to amalgamate the wireless model with a limited power budget with mobility based energy efficient clustering of IoT devices using D2D communication. The literature of IoT data collection using D2D communication lacks a unified approach that is applicable for both static and dynamic IoT devices.Our simulation results depicts that our heuristically developed scheme will successfully reduce the usage of power and system resources.
\end{abstract}

\section{Keywords}

Co-relative mobility; device to device communication; multihop; IoT; data uploading; cluster formation; accelerometer;energy efficiency;less computationally complex;D2D-EE;inte

${ }^{*}$ First author,corresponding author
${ }_{\dagger}$ Second author
${ }_{\ddagger}^{\ddagger}$ Third author
$\S_{\text {Third author }}$

Permission to make digital or hard copies of all or part of this work for personal or classroom use is granted without fee provided that copies are not made or distributed for profit or commercial advantage and that copies bear this notice and the full citation on the first page. To copy otherwise, to republish, to post on servers or to redistribute to lists, requires prior specific permission and/or a fee.

Mobimedia 2017, July 13-14, Chongqing, People's Republic of China Copyright (c) 2017 EAI 978-1-63190-156-0 cluster;stationary nodes;non stationary nodes

\section{INTRODUCTION}

The advent of the internet of thing brings a lot of new opportunities and research avenues for the world. The deluge of cloud-based services and the revolution in IC (integrated chip) fabrication industry that made sensors really cheaper than the past, reinforced the IoT framework. Today IoT has become a favourite research topic among mostly all the top tech companies on the Forbes list of the new startups. This era will be remembered as a stepping stone to a more connected and conscious world where IoT has implementations from space ships to cells of our body. However with great opportunities comes great responsibilities [1], the responsibility to efficiently route the data generated by the myriad of IoT implementations in the physical layer. The coexistence of diverse communication technologies $(3 \mathrm{G} / 4 \mathrm{G} / \mathrm{Wi}-$ Fi/GPRS) at the same time, spare no other option but to find a cooperative technology that can be used as a tool to route data with the help of peers in the proximity. 3rd Generation Partnership Project has defined the aforementioned technology as machine type communication (MTC) [4]. Throughout this paper, we will use the term device to device communication (D2D) instead of MTC. The device to device communication between user equipment (UEs) in proximity will enhance the spectral and power efficiency.

A current estimate has been made by Ericsson [3] that globally at present 10 million devices are connected and a total 50 billion devices are expected to get connected in upcoming five years. To accommodate such vast heterogeneous data in an aggregation model we have identified power budget, dynamicity and computational complexity among UEs as the major challenges. Hence there is a certain need of approaches with reduced computational complexities to cure latency, poor energy efficiency and less dynamic nodes, as key properties. The device densities of the UEs in specific locations have been utilized in the proposed algorithm for D2D communication in mutual proximity, the kernel of the scheme is to cooperatively upload the content of UEs to the remote base station (BS) by forming a multi-hop D2D 
framework. Initially the devices will form clusters having a cluster head at the helm. The cluster heads will form a multi hop path among themselves to upload the data to the BS. Moreover the data will be finally uploaded by that cluster head which is closest to the BS. In this paper, our work has been focused on developing energy efficient and computationally less complex algorithm for IoT data uploading using D2D communication links.

Our major contributions are listed below:

(1) We have identified that due to the limited power budget the use of GPS is not a prudent option. A novel alternative has been ideated to get the velocity of non-stationary UEs. As discussed in section 3.0.2.

(2) The algorithm I in our proposal is a fairly less computationally complex approach to from clusters, this heuristic approach will also save energy as shown in section 4 .

(3) The algorithm II in our proposal will deal with an energy efficient data accumulation and uploading will be based on multihop inter-cluster D2D communication as discussed in section 3.2.

(4) Our approach will accommodate both stationary and non-stationary nodes of IoT devices.

(5) Our approach outperforms the recent research in IoT data collection [2] based on energy efficiency as shown in section 4 .

\section{BACKGROUND AND RELATED WORK}

The Device to device communication was first introduced in 3GPP [6] in release 11 and later to bolster the framework further technical details were published in release 12 . The Proximity communication enabled by D2D has gained huge momentum as a way to overcome the demerits of the conventional cellular system. The major merits of D2D communication are [5]: (a) Low power consumption, due to interaction of devices in mutual proximity that requires less power, (b)transmissions with high data rate due to mutual cooperation among devices, (c) reliable communication, (d) reduced overhead to BS, (e) heterogeneous connectivity of devices, since devices using different technologies such as Wi-Fi, LTE-A and etc, all can be accommodated. D2D can be categorized in two different ways [2] one is implemented using licensed cellular spectrum (inband D2D) and the other uses unlicensed spectrum (outband D2D). The inband communication which seems to be the favourite topic of the research fraternity based on the number of research published can be further classified into two categories: underlay D2D where, cellular and D2D communication share same resources simultaneously whereas in overlay D2D, there are certain dedicated resources provided to D2D to operate. In case of outband D2D it is aimed to eliminate the interference problem, but since BS is not involved in the scenario it needs to rely on interfaces like WiFi, Bluetooth and infrared etc. D2D technology has been read and researched for quite some time and $[7,8]$ has successfully depicted the general idea and modern implementations of the technology.

As already discussed due to the vast number of IoT devices D2D is the favourable candidate to be used in data aggregation as discussed in [9]. A. Orsino et al. in their paper [2] have presented a very interesting scenario of IoT data collection for smart cities, this paper has addressed a wide range of problems with detailed solution. However some assumptions like stationary UEs, round-robin resource allocation etc, made in the aforementioned research limited the scope of solution. G. Rigazzi et al. have also proposed a good alternative resource allocation scheme in [10] that is both practical and easily implementable. Militano et al. in the paper [9] have presented a cooperative coalition formation based on social trust and proximity. Data aggregation can also be used to efficiently manage the resource block (RBs) and power, the literature of this technology is vast. Moreover, a good taxonomy of data aggregation can be found in [14] in which Schubert et al. have proposed a novel data aggregation technique for IoT devices, they used data aggregation as a tool to reduce the power consumption. In general we have observed that the wireless research fraternity is trying to deal the IoT data transmission the same way as they dealt with wireless sensor networks data transmission. However, IoT data transmission has certain differences with wireless sensor network (WSN) data transmission: a) the content generated by IoT devices are relatively smaller than general WSN data, (b) generally the channel is expected to be good for data transmission in WSN but in IoT it doesn't make much difference, (c) in IoT data transmission the system latency should be minimum unlike WSN where generally there is no such obligation. Finally, the aforementioned related works are focused only on reducing power consumption of IoT data transmission however, our proposed solution will discuss a practical tradeoff between computational complexity and power consumption in IoT data transmission using D2D communication.

\section{SYSTEM MODEL}

The proposed system is formulated using Long Term Evolution Advanced (LTE-A) network, which in case provide dedicated resources for D2D communication i.e. the system follows inband overlay D2D communication protocol. To maintain the simplicity throughout the section we will demonstrate our proposal using a single cell scenario as shown in figure 1. Following the standard procedure of data uploading in the cellular network, primarily the BS will collect the cell-mode CQI (Channel quality indicator) feedbacks from all IoT devices (nodes) willing to upload some data. Secondarily the BS will collect the D2D mode CQI values and will form a D2D CQI matrix (DCM) [2].Suppose each node $n_{i}=\left\{n_{1, \ldots \ldots,}, n_{\left|n_{i}\right|}\right\} \in N$ is a part of cluster $s_{i}=\left\{s_{1}, \ldots \ldots, s_{\left|s_{i}\right|}\right\} \in S$ in a cell governed by a BS.Both the stationary and non-stationary IoT devices (nodes) will broadcast beacon messages to find a 1-hop neighbour. Here we will use a range based method to evaluate the angle of arrival (AOA) and received signal strength indicator (RSSI) [13] to locate the distance of an unknown neighbouring node and angle w.r.t the reference node (node which needs a relay node to transfer data). The co-relative mobility between nodes will be calculated and stored in each node as a matrix. Next, all the nodes will choose a 1-hop neighbour node based on co-relative mobility among the neighbouring to relay their data. This process when implemented by all nodes in a cell, the nodes group among themselves to from dynamic or static clusters.

We assume that each device is a smart device with either accelerometer or GPS mounted inside it. Based on the underline principle of inband overlay device to device communication BS will provide dedicated resources. Moreover using the DCM (D2D CQI matrix) mentioned in the previous section, BS will monitor and control entire D2D scenario. A link that is not feasible as per the DCM will not be con- 
nected. As the algorithm runs the BS will allot a node id to each node and will make rings of nodes based on the distance from it. Then, each node in the cell will broadcast beacons to locate a relay node in its proximity. A relay node is an IoT device which will help to remote IoT nodes to upload their data cooperatively to the BS using D2D links. Here we will use a range based method to evaluate the angle of arrival (AOA) and received signal strength indicator (RSSI) [15] to locate the distance of an unknown neighbouring node and angle w.r.t the reference node (node which needs a relay node to transfer data). Each node will compute a dynamic $\mathrm{N} \times \mathrm{N}$ matrix which will be similar to DCM matrix however instead of CQI value it will contain co-relative mobility of reference node w.r.t all other nodes. This matrix will further play a crucial role in searching a deserving neighbouring $\mathrm{n}$ ode to relay data. We have also assumed that all the UEs are smart devices and can send or receive data autonomously. Prior moving to the main proposal we need to define certain keys concepts which will be used throughout the proposal.

\subsubsection{Energy efficiency}

Inefficient use of any resources is equivalent to wasting of resources. We have focused all our efforts to optimise the energy efficiency with the computational complexity, which in case is a trade-off between these two parameters in this paper. Moreover, the amount of energy to be used for our proposal will be based on the number of packets to be uploaded by each node. Since energy efficiency $(\eta)$ have been researched for a long time we have used the standard equation [2] from the literature. The energy efficiency $(\eta)$ of the system which has $\mathrm{N}$ number of users can be computed as:

$$
\eta=\sum_{N} \frac{d_{N}}{E_{N} \cdot r_{N} \cdot T T I}
$$

Where $d_{N}$ is the total volume of data(in bits) to be uploaded, $E_{N}$ is the average power consumed to deliver a single packet of data, $r_{N}$ is total number of data packets to be uploaded by all the nodes and TTI is transmission time interval which is fixed for all packets.

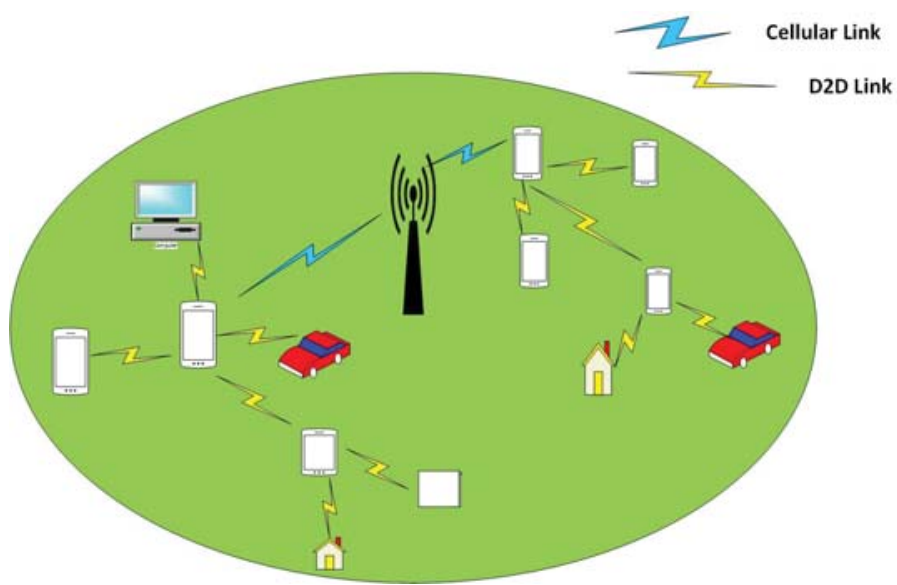

Figure 1: Multi hop D2D content uploading.

\subsubsection{Accelerometer}

Accelerometer [11] is a device that measures the acceleration of a device, all our smart devices are equipped with it.
A 3 axis accelerometer (most famous) in mobile device provides the $\mathrm{X}, \mathrm{Y}, \mathrm{Z}$ coordinate values, which is used to measure the position and acceleration of the device. The rotation, direction and position are measured using gyroscope sensors present in mostly all smartphones. The acceleration values provided by the device includes gravity as well. We are interested in linear acceleration which does not include gravity. Therefore the accelerometer reading will be passed to filters to redefine the results to be used by our proposal. The function that calculates velocity is shown below.

$$
v(t)=v(0)+\sum a \times \delta t
$$

Where $\mathrm{v}(\mathrm{t})$ is the instantaneous velocity at time $\mathrm{t}, \mathrm{v}(0)$ is the initial velocity, a is the instantaneous acceleration at time $\mathrm{t}$ and $\delta t$ is the time takes to accelerate. Here we are more interested in the horizontal component of velocity of nodes. The horizontal component of velocity of node $n_{x}$.

$$
V_{x}(t) \cos \theta
$$

where $\theta$ is the angle of velocity w.r.t other node Since our proposal is focused on the reduction of power consumption, which in case increases the device battery lifecycle. We are proposing to the use of accelerometers as speedometers to calculate the velocity of IoT nodes except vehicles. Our proposal also includes vehicles, they may use the GPS because they have a constant source of power and it gets charged while moving. This will save us energy and computation$\mathrm{s}$ used by GPS to get the position coordinates and then compute the velocity based on movement. The accelerometer readings may not be accurate but since our proposal deals with the relative velocity with respect to the other vehicles, it will suffice the purpose.

\subsubsection{Co-relative mobility}

We know relative mobility [12] has a vast literature and research fraternity have used the term relative mobility in fairly different scenarios. Moreover in this proposed scheme the term co-relative mobility has been used as a function i.e. cross correlation between relative mobility between two nodes $n_{x}$ and $n_{y}$ where $x, y \in i$ and $n_{x}, n_{y} \in N$ at time t. As already mentioned to calculate the distance between two nodes, we will use the RSSI and AOA techniques. Distance between two nodes $n_{x}$ and $n_{y}$ where $x, y \in i$ and $n_{x}, n_{y} \in N$ at time $\mathrm{t}$ can be defined as the $D_{X Y}(t)$.

The normalized distance $\bar{D}_{X Y}(t)$ can be defined as:

$$
\bar{D}_{X Y}(t)=D_{X Y} / C R
$$

Where $\mathrm{CR}$ is the maximum communication range of the beacon.

The horizontal velocity of nodes can be defined as:

$$
V_{x}(t) \cos \theta
$$

$$
V_{y}(t) \cos \theta
$$

Here $\theta$ is the velocity vector angle, moreover the orientation of the movement w.r.t the reference node can be measured by $\theta$.

$$
\bar{V}_{x}(t)=\left|V_{x}(t) \cos \theta\right|
$$


Where $\bar{V}_{x}(t)$ is only the magnitude of the horizontal component of velocity of node $n_{x}$.

$$
R M_{X}(t)=\alpha \bar{D}_{X Y}(t)+\beta V_{X}(t)
$$

Here, $R M_{X}(t)$ is the relative mobility of node $n_{x}$. The values of $\alpha$ and $\beta$ are subjected to change based on traffic scenarios.

Using eqs. 4,7 and 8 the co-relative mobility between node $\mathrm{X}$ and node $\mathrm{Y}$ can be defined as the cross correlation between the relative mobility of two nodes as shown below:

$$
\operatorname{cr}_{X Y}(t)=\text { Correlation }\left(R M_{X}(t), R M_{Y}(t)\right)
$$

Here, $\operatorname{cr}_{X Y}(t)$ is the co-relative mobility of two nodes $n_{x}$ and $n_{y}$ where $x, y \in i$ and $n_{x}, n_{y} \in N$ at time $\mathrm{t}$ and $\mathrm{n}$ is the total number of nodes.

\subsection{Proposed cluster formation scheme}

The formation of cluster can be summarized in the following steps:

Step1: The BS will randomly allocate a unique node id to all the nodes.

Step2: The base station which is assumed to be at the centre of the cell will group the nodes based on the distance from the centre. The BS will form several circular rings based on the radial distance from itself. The rings will be named starting from the closest to the farthest distance from the BS. Such as the ring which arrives at the first place when we start from the BS along it's radius to the outward direction will be named as ring $\mathrm{k}$. Where $\mathrm{k}$ is the total number of rings formed. Each node will store the aforementioned number as it's own ring id.

Step3: At this point, all the nodes (reference nodes) will start broadcasting advertisement messages within their threshold perimeter. Simultaneously the neighbouring nodes which fall inside the threshold radius will compute the co-relative mobility based on equation 9 w.r.t the advertiser node.

Step4: The neighbouring node (current node) which might have received a lot of advertisement messages will calculate the competency of the reference nodes (advertiser node) to join a cluster based on the relative mobility. The neighbouring node will store this competency value of each advertiser nodes in an array known as competency array. This array contains the competency value of all the nodes that has sent advertisement message to the current node.

Step5: The neighbouring node (current node) will join the cluster of that one node which has the highest co-relative mobility among the other advertiser nodes, which in case will provide the highest competency to the advertiser node. An important constraint imposed on the selection by the current node is that it can only select a reference node which has a ring id equal to it's own ring id.

Step6: When the aforementioned process is implemented over a large group of nodes they will form several variable size clusters. As presented in our simulation studies.

The aforementioned process, can be easily understood using the flowchart shown in figure 2. The following section will depict our idea of data uploading to BS based on the inter-cluster multi hop data transmission.

\subsection{Proposed data uploading scheme}

As already mentioned the data size generated by the IoT devices are relatively small, thus it may not require a high

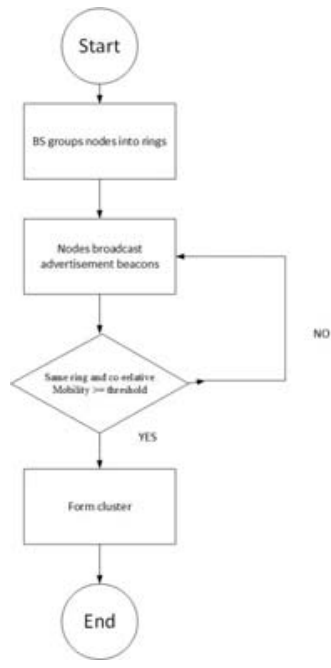

Figure 2: Flow of cluster formation scheme.

CQI, taking advantage of such traits of IoT data we have formed a multi hop data routing from CM to the BS(sink). Moreover only the $\mathrm{CH}(\mathrm{s})$ of clusters will participate in the multi hop data transmission, in that case it is an inter cluster head data transmission.

Post cluster formation all the CMs will upload their data to the $\mathrm{CH}$ using D2D links. A CH will aggregate all the data uploaded by the CMs and start looking for next hop CH to relay the data in the adjacent ring closer to the BS.

The following steps will summarize the proposed data $\mathrm{u}-$ ploading scheme:

Step1: The CM(s) will upload the data to the CH (current $\mathrm{CH})$ of a particular cluster.

Step2: The current $\mathrm{CH}$ will aggregate the data and will broadcast advertisement messages to find potential next hop $\mathrm{CH}$ in the adjacent ring closer to the BS.

Step3: The interested potential relay nodes will acknowledge for the advertisement messages to the current $\mathrm{CH}$.

Step4: The current $\mathrm{CH}$ will select a next hop $\mathrm{CH}^{1}$ which as highest co-relative mobility with itself.

Step5: The above mentioned steps will be repeated until all the data packets reached the BS (sink).

The steps of the data uploading scheme is presented in the form of a flowchart in figure 3 .

\section{IMPLEMENTATION AND RESULTS}

Our proposed D2D based IoT data uploading scheme is supported by experiments, designed in Matlab 2012. To judge the competitive performance of our proposal with other D2D based IoT data collection schemes we compared our findings with the findings of the recently published research,known as D2D-EE scheme. The D2D-EE scheme which is proposed in [2] is also based on D2D communications within the cluster of devices and aggregates the data in cluster heads before uploading it to BS, due to the similar nature w.r.t our proposal it is found suitable as an alternative solution hence, comparisons will be interesting. During the simulative study, we have fabricated a system which consists of $\mathrm{N}$ random nodes and a BS at the centre.

${ }^{1}$ All the CHs will forward it's own data before, relaying other's data. 


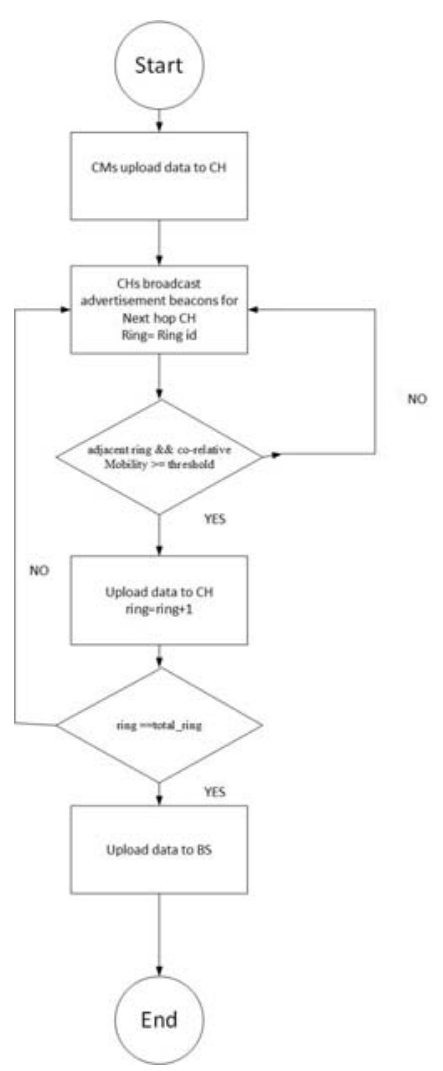

Figure 3: Flow of data uploading scheme.

We have chosen energy efficiency and computational complexity as the performance parameters. However currently our simulation results are based on energy efficiency. There are some parameters that were kept constant while performing the experiments as shown in table 1 . The formation of clusters based on co-relative mobility can be seen in figure 4 .

Table 1: Simulation parameters

\begin{tabular}{lc}
\hline Parameter & Value \\
\hline$\alpha$ & 0.5 \\
$\beta$ & 0.5 \\
Beacon transmission range & $300 \mathrm{~m}$ \\
Transmission time interval (TTI) & $1 \mathrm{~ms}$ \\
Control packet length & $200 \mathrm{bits}$ \\
Initial energy of each node & 0.5 joule \\
Maximum number of rings & 5 \\
\hline
\end{tabular}

During the first experiment, we have plotted the energy efficiency which is expressed in bits/joule while varying the no. of IoT devices from 50 to 500 and the packet length is fixed at 10 bytes, as shown in figure 5 . AS depicted in the plot the energy efficiency is increasing with increasing number of devices for both the schemes. Moreover, the proposed solution is highly superior in all the cases and it is approximately five times more energy efficient than the D2D-EE scheme based on number of devices taking part.

During the second experiment, we have plotted the energy

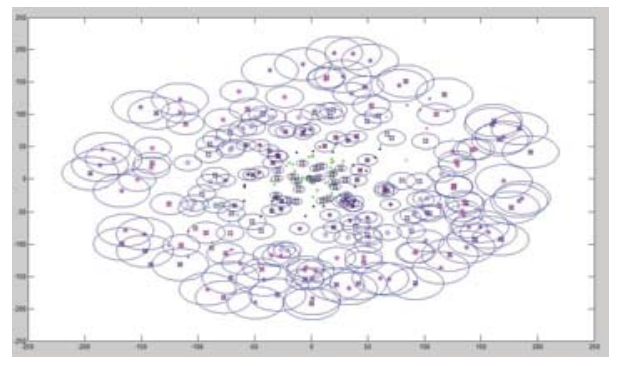

Figure 4: Formation of simulated clusters based on corelative mobility.

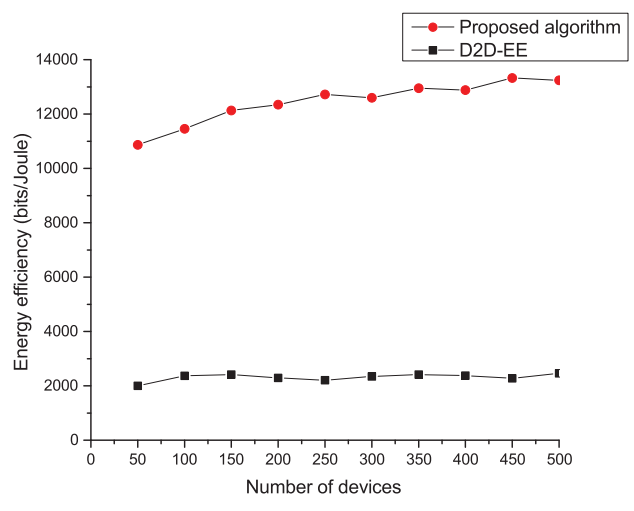

Figure 5: Energy efficiency vs. Number of devices.

efficiency while varying the packet size from 0 to 100 bytes while keeping the number of devices fixed at 50, as shown in figure 6. AS depicted over the plot the energy efficiency of our proposed solution is increasing till 40 bytes to attain the maximum energy efficiency and after that is became abrupt w.r.t it's preceding portion. In the case of the D2D-EE solution, it increases with increase in packet size.Moreover, our proposed solution retained its supremacy over D2D-EE solution in nearly 80 percent of the experimental outputs.

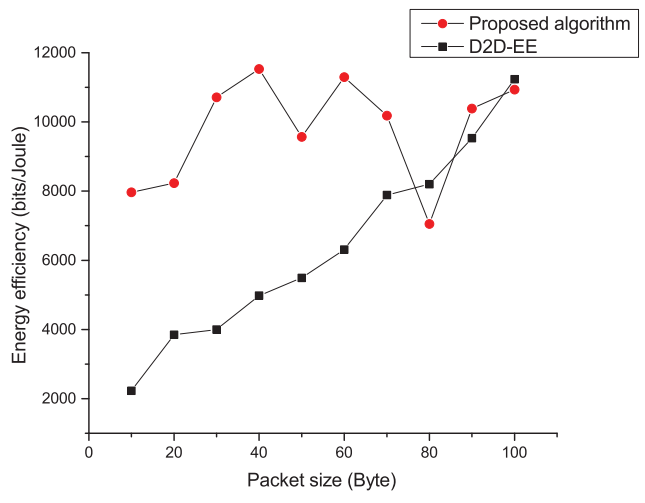

Figure 6: Energy efficiency vs. Packet size.

\section{FUTURE WORK}

During the present research, we have identified that both computational complexity and energy efficiency are crucial 
to IoT data uploading based on D2D inband overlay communication links. The simulation of computational complexity will be carried out during the extension of this research to improve our proposal. Moreover, a practical resource allocation scheme based on the rings to support our current system will also be simulated in the upcoming research.

\section{CONCLUSIONS}

In this paper, an IoT data uploading scheme using D2D communication has been proposed, the proposal consist of two main ideas which are cluster formation and data routing schemes. This proposal can accommodate both stationary and non-stationary nodes. The cluster formation scheme uses a novel idea of co-relative mobility to group nodes into variable size clusters. The routing of data consists of inter-cluster multihop data relaying which adds to our main agenda that is energy efficiency. The simulation study shows that the proposal is highly energy efficient and can handle small as well as large number of nodes. Our proposal is suitable for scenarios where the solution is expected to be less computationally complex as well as high energy efficient to collect IoT data using D2D links.

\section{ACKNOWLEDGMENTS}

This work is supported by the National Natural Science Foundation of China (61371097), Program for Innovation Team Building at Institutions of Higher Education in Chongqing (CXTDX201601020).

\section{REFERENCES}

[1] "Spider Man marvel movie 2002," Marvel studios.

[2] Antonio Orsino,G.Araniti, L. Militano, et al, "Energy efficient IoT data collection in smart cities Exploiting D2D Communications," MDPI Sensors, vol. 16,pp. 1-19,2016.

[3] Ericsson, "More than 50 Billions Connected Devices," Ericsson, vol. 1,Stockholm, Sweden,Feb. 2011.

[4] 3GPP, "TS 22.368, "Service Requirements for Machine-Type Communications(MTC)," Tech. Rep., vol. 13, no. 1,Dec. 2014.

[5] B. Zhou, H. Hu, S.-Q. Huang, and H.-H. Chen,"Intracluster deviceto-device relay algorithm with optimal resource utilization," IEEE Transactions on Vehicular Technology, vol. 62, no. 5, pp. 2315Ü2326, Nov. 2013.

[6] 3GPP, "TS 22.803, "Feasibility Study for Proximity Services (ProSe)," 3GPP: Sophia Antipolis Cedex, Rel. 12,France,Dec. 2014.

[7] A. Asadi,Q. Wang,V.Mancuso,"A Survey on Device-to-Device Communication in Cellular Networks," IEEE Communication Surveys and Tutorials, vol. 16, no. 5, pp. 1801Ü1819, Apr. 2014.

[8] Y.Cao, T.Jiang,C.Wang,"Cooperative device to device communications in cellular networks," IEEE Wireless Communications,pp. 124-129, Jun. 2015.

[9] L. Militano, et al, "A. Device-to-Device Communications for $5 \mathrm{G}$ Internet of Things," EAI Endorsed Trans. Internet Things 2015, vol. 15, Oct. 2015.

[10] Rigazzi G., Chiti F., Fantacci R., Carlini C. "Multi-hop D2D networking and resource management scheme for M2M communications over LTE-A systems," In Proceedings of the 2014 InternationalWireless Communications and Mobile Computing Conference (IWCMC), Nicosia, Cyprus, pp. 973Ü978, Aug. 2014.

[11] https://en.wikipedia.org/wiki/Accelerometer.

[12] M. Azizian, S. Cherkaoui, A.S. Hafid, "A Distributed D-hop Cluster Formation for VANET," In Proceedings of International Wireless Communications and Mobile Computing Conference (IWCMC), 2014.

[13] N. Patwari, J.N. Ash, S. Kyperountas, "Locating the nodes," IEEE Signal Processing Magazine, vol. 22, pp. 54-69, Apr. 2014.

[14] K. Schubert, N.Bambos,"Data aggregation for Low Power Wireless Devices," In Proceedings of IEEE Milcom Conference), track.5, Dec. 2016. 